\title{
Investigation of a CDDW Hamiltonian to Explore Possibility of Magneto-Quantum Oscillations in Electronic Specific Heat of Hole-Doped Cuprates
}

\author{
Partha Goswami ${ }^{1}$ and Manju Rani ${ }^{1,2}$ \\ ${ }^{1}$ D.B.College, University of Delhi, Kalkaji, New Delhi 110019, India \\ ${ }^{2}$ Department of Electronic Science, University of Delhi South Campus, New Delhi 110021, India
}

Correspondence should be addressed to Partha Goswami, physics_goswami@yahoo.co.in

Received 3 February 2010; Revised 31 July 2010; Accepted 25 August 2010

Academic Editor: P. Guptasarma

Copyright (C) 2010 P. Goswami and M. Rani. This is an open access article distributed under the Creative Commons Attribution License, which permits unrestricted use, distribution, and reproduction in any medium, provided the original work is properly cited.

\begin{abstract}
We investigate a chiral d-density wave (CDDW) mean field model Hamiltonian in the momentum space suitable for the hole-doped cuprates, such as YBCO, in the pseudogap phase to obtain the Fermi surface (FS) topologies, including the anisotropy parameter $(\dot{\varepsilon})$ and the elastic scattering by disorder potential $\left(\left|v_{0}\right|\right)$. For $\dot{\varepsilon}=0$, with the chemical potential $\mu=-0.27 \mathrm{eV}$ for $10 \%$ doping level and $\left|v_{0}\right| \geq|t|$ (where $|t|=0.25 \mathrm{eV}$ is the first neighbor hopping), at zero/non-zero magnetic field $(B)$, the FS on the first Brillouin zone is found to correspond to electron pockets around antinodal regions and barely visible patches around nodal regions. For $\dot{\varepsilon} \neq 0$, we find Pomeranchuk distortion of FS. We next relate our findings regarding FS to the magneto-quantum oscillations in the electronic specific heat. Since the nodal quasiparticle energy values for $B=0$ are found to be greater than $\mu$ for $\left|v_{0}\right| \geq|t|$, the origin of the oscillations for nonzero $B$ corresponds to the Fermi pockets around antinodal regions. The oscillations are shown to take place in the weak disorder regime $\left(\left|v_{0}\right|=0.25 \mathrm{eV}\right)$ only.
\end{abstract}

\section{Introduction}

In the recent past, extensive theoretical [1-9] and experimental efforts [10-20] have been carried out to understand the pseudogap (PG) phenomenon in the normal state of the underdoped cuprates. These enterprises have led to substantial understanding of the Fermi surface (FS) topologies of the material. The noteworthy aspect of the PG state of the hole-doped cuprates, such as YBCO, is that it could be characterized with a variety of coexisting/competing orders, as have been reported by previous workers $[1-11,21]$. The theorists [1-9] among them have put forward explanations of the (experimentally) observed facts/anomalies [11-20,22] in various physical properties, which carry the signature of the complexities in PG state, with high degree of success. In this communication, starting with a chiral d-density wave (CDDW) $[1,2]$ mean field Hamiltonian $H_{d+i d}$ in momentum space involving suitable dispersion with hopping integrals $\left(t_{i j}\right)$ and incorporating the effect of elastic scattering by disorder potential $\left(\left|v_{0}\right|\right)$ in the Fourier coefficient of singleparticle Green's function (defined by $H_{d+i d}$ ), we show that the reasonably well-developed Fermi pockets in the antinodal regions and barely visible (hole-like) patches in the nodal regions of FS, at zero/nonzero magnetic field, can be obtained from the energy eigenmodes of the matrix in $H_{d+i d}$ for $\left|v_{0}\right| \geq|t|$ and $\left|t^{\prime} / t\right|=0.4$ where $t(=0.25 \mathrm{eV})$ and $t^{\prime}$, respectively, are the first neighbor and the second neighbor hopping integrals. It must be mentioned that the appearance of the pockets depends crucially on the ratio $\left|t^{\prime} / t\right|$ (see Figure 2(e)). The angle resolved photo-emission spectroscpic (ARPES) studies [23-28] (including the vacuum ultraviolet (VUV) laser-based ARPES [29]) of cuprates, where the experimental observations roughly correspond to the socalled "maximal intensity surface" explained in [25], have not shown the evidence of the existence of the Fermi pockets so far. Doiron-Leyraud et al. [13-15], however, have 
detected quantum oscillations in the electrical resistance of underdoped YBCO establishing the existence of a welldefined FS with Fermi pockets in the antinodal region when the superconductivity is suppressed by a magnetic field. Furthermore, Riggs et al. [12] have observed the oscillations in the specific heat of YBCO-Ortho II samples (in the presence of a magnetic field $B=45 \mathrm{~T}$ ), the same type YBCO samples were investigated by Doiron-Leyraud et al. [13-15], confirming the findings regarding Fermi pockets. The aims of the present theoretical investigation are (i) to show the existence of Fermi pockets in the antinodal regions and (ii) to explore the possibility of approximate $(1 / B)$-oscillations in the specific heat in order to verify the finding of Riggs et al. [12]. While we succeed in showing the existence of the Fermi pockets (see Figures 2 and 3 ) for the weak $\left(\left|v_{0}\right|=0.25 \mathrm{eV}\right)$ as well as the strong disorder $\left(\left|v_{0}\right|=1 \mathrm{eV}\right)$ regimes, the quantum oscillations are shown to be possible for $B \geq 17 \mathrm{~T}$ in the weak disorder regime only. Our reason for the identification of the PG state with the CDDW state rather than the well-known $[3,4]$ d-density wave (DDW) state is that the CDDW ordering offers a theoretical explanation [5] of the nonzero polar Kerr effect observed recently in YBCO by Xia et al. [11]. Also, since the experimental signature of nematic order has been observed recently in cuprates in neutron scattering experiments [10], it is felt that the hopping anisotropy [30], which captures the electronic nematicity at the mean-field level, ought to be a part of the ongoing investigation to be dealt with in future.

The paper is organized as follows. In Section 2 we present the mean field Hamiltonian $H_{d+i d}$ in momentum space involving in-plane hopping anisotropy. In Section 3 we discuss the elastic scattering by impurities and relate it to the issue of the Fermi pockets as this occupies the centre stage [31] in the magneto-quantum oscillation context. In Section 4 we discuss the issue of the magneto-quantum oscillations in electronic specific heat in detail. The paper ends in Section 5 with the concluding remarks.

\section{Model Hamiltonian}

For a magnetic field applied in $z$-direction, we consider a normal state tight-binding energy dispersion involving $t, t^{\prime}$, and $t^{\prime \prime}$ which are, respectively, the hopping elements between the nearest, next nearest (NN), and NNN neighbours:

$$
\begin{aligned}
\varepsilon_{k, N}(B)= & -2\left(t_{x} \cos \left(k_{x} a\right)+t_{y} \cos \left(k_{y} a+\varphi\right)\right) \\
& +4 t^{\prime} \cos \left(k_{x} a\right) \cos \left(k_{y} a+\frac{\varphi}{2}\right) \\
& -2\left(t_{x}^{\prime \prime} \cos 2 k_{x} a+t_{y}^{\prime \prime} \cos 2\left(k_{y} a+\varphi\right)\right) \\
& +\hbar\left(N+\left(\frac{1}{2}\right)\right) \omega_{c} .
\end{aligned}
$$

Here the Landau level(LL) index $N=0,1, \ldots$ and " $a$ " is the lattice constant (of YBCO). The vector potential $\mathbf{A}$ is assumed to be in the Landau gauge: $\mathbf{A}=\left(\begin{array}{lll}0-B x & 0\end{array}\right)$. The quantity $\omega_{c}=\mathrm{eB} / \mathrm{m}^{*}$ is the cyclotron frequency where $\mathrm{m}^{*}$ is the effective mass of the electrons. The Zeeman splitting has not been taken into account. Following Hackl and Vojta [30], we have introduced a hopping anisotropy parameter $\dot{\varepsilon}$, such that the hopping elements obey $t_{x, y}=(1 \pm \dot{\varepsilon} / 2) t$ and $t_{x, y}^{\prime \prime}=(1 \pm \varepsilon / 2) t^{\prime \prime}$. For $\dot{\varepsilon} \neq 0$, the lattice rotation symmetry is spontaneously broken. In the numerical calculations below we take $t$ as an energy unit, where $t=0.25 \mathrm{eV}, t^{\prime}=0.4 t$, and $t^{\prime \prime}=0.0444 t$. These values are the same as in [3]. Furthermore, we shall consider the value of the chemical potential $(\mu)$ of the fermion number to be $(-0.27 \mathrm{eV})$ for $10 \%$ hole doping [3]. In the presence of a vector potential $\mathbf{A}$, the hopping amplitudes $t_{i j}$, corresponding to the sites $\mathbf{i}$ and $\mathbf{j}$, assume the form $\left[t_{i j} \exp \left(a_{i j}\right)\right]$, where $a_{i j}=$ $\left(\pi / \Phi_{0}\right) \int_{j}^{i}$ A.dl and $\Phi_{0}=(h / 2 e)$. For the first neighbor hopping, say, corresponding to the sites $\mathbf{i}=(a, 0)$ and $\mathbf{j}=(a, a)$, the quantity $a_{i j}=-\left(\pi / \Phi_{0}\right) \int_{(a, a)}^{(a, 0)} B x d y=\varphi$, where $\varphi=\left(2 \pi e B a^{2} / h\right)$, is the Peierls phase factor. Similarly, for the second neighbor hopping, say, corresponding to the sites $\mathbf{i}=(a, 0)$ and $\mathbf{j}=(2 a, a)$, the quantity $a_{i j}=$ $-\left(\pi / \Phi_{0}\right) \int_{(2 a, a)}^{(a, 0)} B y d y=\varphi / 2$. These explain the reason behind the appearance of $\varphi$ and $\varphi / 2$, respectively, in the first and the second terms of (1). Likewise, the reason for the appearance of $\varphi$ in the third term of (1) could be explained.

In the second quantized notation, the Hamiltonian (with index $j=(1,2)$ below corresponding to two layers of YBCO) for the chiral $d+i d$ density-wave state, together with the anisotropy in the hopping parameters, in the presence of magnetic field $(B)$ can be expressed as

$$
H_{d+i d}(B)=\sum_{k, N, \sigma, i=1,2} \Phi_{k, \sigma}^{(i) \dagger} E_{N}(k, B) \Phi_{k, \sigma}^{(i)},
$$

where $\Phi_{k, \sigma}^{(i) \dagger}=\left(d_{k, \sigma}^{\dagger(1)} d_{k+Q, \sigma}^{\dagger(1)} d_{k, \sigma}^{(2) \dagger} d_{k+Q, \sigma}^{\dagger(2)}\right)$ and $E_{N}(k, B)=$ $\left[\varepsilon_{k, N}^{U}(B) I_{4 \times 4}+\zeta_{k}(B) \cdot \boldsymbol{\alpha}\right]$. Here $\boldsymbol{\alpha}=\left(\begin{array}{llll}\alpha_{1} & \alpha_{2} & \alpha_{3} & \alpha_{4}\end{array}\right)$ with

$$
\alpha_{i}=\left(\begin{array}{cc}
\sigma_{i} & 0 \\
0 & \sigma_{i}
\end{array}\right) \quad(i=1,2,3), \quad \alpha_{4}=\left(\begin{array}{cc}
0 & I_{2 \times 2} \\
I_{2 \times 2} & 0
\end{array}\right),
$$

$I_{4 \times 4}$ and $I_{2 \times 2}$, respectively, are the $4 \times 4$ and $2 \times 2$ unit matrices, $\sigma_{i}$ are the Pauli matrices, and $\zeta_{k}(B)=\left(-\chi_{k}-\Delta_{k} \varepsilon_{k}^{L}(B) t_{k}\right)-$ a four-component vector. Here the chiral order parameter [1], $D_{k} \exp \left(i \theta_{k}\right)$, is given by $D_{k}=\left(\chi_{k}^{2}+\triangle_{k}^{2}\right)^{1 / 2}$ and $\cot \theta_{k}=$ $\left(-\chi_{k} / \triangle_{k}\right)$ with

$$
\begin{aligned}
\chi_{k} & =-\left(\frac{\chi_{0}}{2}\right) \sin \left(k_{x} a\right) \sin \left(k_{y} a\right), \\
\triangle_{k} & =\left(\frac{\triangle_{0}(T)}{2}\right)\left(\cos k_{x} a-\cos k_{y} a\right) .
\end{aligned}
$$

We consider the simplest form of the modulation vector, $\mathbf{Q}=( \pm \pi, \pm \pi)$. The quantity $\mathrm{t}_{k}$ is momentum conserving tunneling matrix element which for the tetragonal structure of cuprates is given by $t_{k}=\left(t_{0} / 4\right)\left(\cos k_{x} a--\cos k_{y} a\right)^{2}$. As in [3], we take $t_{0}=0.032 t$. The reader may note that in (2) we have disregarded the LL mixing completely. The energy eigenvalues of $E_{N}(k, B)$ are

$$
E_{N}^{(j, v)}(k, B)=\left[\varepsilon_{k, N}^{U}(B)+j w_{k, N}(B)+v t_{k}\right],
$$


where $\varepsilon_{k, N}^{U}(B)=\left(\varepsilon_{k, N}(B)+\varepsilon_{k+Q, N}(B)\right) / 2, \varepsilon_{k, N}^{L}(B)=$ $\left(\varepsilon_{k, N}(B)-\varepsilon_{k+Q, N}(B)\right) / 2$ and $w_{k, N}(B)=\left[\left(\varepsilon_{k, N}^{L}(B)\right)^{2}+D_{k}^{2}\right]^{1 / 2}$. Here $j$ is equal to $( \pm 1)$ with $j=+1$ corresponding to the upper branch $(\mathrm{U})$ and $j=-1$ to the lower branch $(\mathrm{L})$; for a given $j, v= \pm 1$. For LL index $N=0,1$, we shall have twofold splitting of $E_{N}^{(j, v)}(k, B)$ for a given $(j, v)$. With these eigenvalues, we obtain the noninteracting Matsubara propagator

$$
\begin{aligned}
G_{N}\left(k, \omega_{n}\right)=\sum_{\nu= \pm 1}\{ & V_{k, N}^{(U, v) 2}\left(i \omega_{n}-E_{N}^{(U, v)}(k, B)\right)^{-1} \\
& \left.+V_{k, N}^{(L, v) 2}\left(i \omega_{n}-E_{N}^{(L, v)}(k, B)\right)^{-1}\right\} .
\end{aligned}
$$

The quasiparticle coherence factors $\left(V_{k, N}^{(U, v) 2}, V_{k, N}^{(L, v) 2}\right)$ are given by the expressions

$$
\begin{aligned}
V_{k, N}^{(U, v) 2} & =\left(\frac{1}{4}\right)\left[1+\left(\frac{\varepsilon_{k, N}^{L}}{w_{k, N}}\right)\right], \\
V_{k, N}^{(L, v) 2} & =\left(\frac{1}{4}\right)\left[1-\left(\frac{\varepsilon_{k, N}^{L}}{w_{k, N}}\right)\right] .
\end{aligned}
$$

The magnetic field dependence of these factors arises through $\varepsilon_{k, N}^{L}(B)$. At hole doping level $\sim 10 \%$, we find that the pseudogap (PG) temperature $T^{*} \sim 155 \mathrm{~K}$. We have assumed the value $\Delta_{0}\left(T<T^{*}\right)=0.0825 \mathrm{eV}=0.3300 t$ in the vicinity of $T^{*}$ and $\left(\chi_{0} / \triangle_{0}\left(T<T^{*}\right)\right)^{2}=0.0025$. We shall now consider the effect of the elastic scattering by impurities on the Fermi surface topology and search for the Fermi pockets. This is an important issue as without these pockets the Onsager relation [31] does not allow one to investigate magneto-quantum oscillations. The impurity potential/disorder with finite range not only has drastic effects on the Fermi surface (FS) topology but also will be seen to affect the density of states at Fermi energy, relevant for the calculation of physical properties, in the following sections.

\section{Elastic Scattering by Impurities and Fermi Energy Density of States}

The effect of elastic scattering by impurities involves the calculation of self-energy $\Sigma\left(\mathbf{k}, \omega_{n}\right)$, in terms of the momentum and the Matsubara frequencies $\omega_{n}$, which alters the single-particle excitation spectrum in a fundamental way. A few diagrams contributing to the self-energy are shown in Figure 1. The wiggly lines carry momentum but no energy as the scattering is assumed to be elastic. The total momentum entering each impurity vertex, depicted by a slim ellipse, is zero. We assume that impurities are alike, distributed randomly, and contributea potential term $V(\mathbf{r}=\mathbf{R}+z \mathbf{k})=$ $\sum_{i} u\left(\mathbf{r}--\mathbf{r}_{i}\right)$, where $u\left(\mathbf{r}-\mathbf{r}_{i}\right)$ is the potential due to a single impurity at $\mathbf{r}_{i}=\mathbf{R}_{i}+z \mathbf{k}$ for a given $z$ and $\mathbf{R}=$ $x \mathbf{i}+y \mathbf{j}$. The potential term $u\left(\mathbf{r}-\mathbf{r}_{i}\right)$ is expanded in a Fourier series $V(\mathbf{r})=\sum_{q, i} V(|\mathbf{q}|) \exp \left[i \mathbf{q} \cdot\left(\mathbf{r}--\mathbf{r}_{i}\right)\right]$. We first consider only the contribution of Figure 1(a). Assuming the scattering by impurities weak, as in [32], we may write it as $\Sigma^{(1)}\left(\mathbf{k}, \omega_{n}\right)=N_{j} \Sigma_{k^{\prime}}\left|V\left(\mathbf{k}-\mathbf{k}^{\prime}\right)\right|^{2} G_{N}\left(\mathbf{k}^{\prime}, \omega_{n}\right)=\sum_{0}^{(1)}\left(\mathbf{k}, \omega_{n}\right)+$ $\Sigma_{e}$ where

$$
\begin{gathered}
\Sigma_{0}^{(1)}\left(\mathbf{k}, \omega_{n}\right)=-N_{j} \sum_{k^{\prime}, \nu= \pm 1}\left|V\left(\mathbf{k}-\mathbf{k}^{\prime}\right)\right|^{2}\left(i \omega_{n}\right) \int_{-\infty}^{+\infty} d \varepsilon \rho(\varepsilon) \\
\times\left[V_{k, N}^{(U, v) 2}\left(\omega_{n}^{2}+E_{N}^{(U, v)}(k, B)^{2}\right)^{-1}\right. \\
\left.\quad+V_{k, N}^{(L, v) 2}\left(\omega_{n}^{2}+E_{N}^{(L, v)}(k, B)^{2}\right)^{-1}\right],
\end{gathered}
$$

$N_{j}$ is the impurity concentration, $V\left(\mathbf{k}-\mathbf{k}^{\prime}\right)$ characterizes the momentum-dependent impurity potential, and $\Sigma_{e}$ is the part of the first-order contribution which can be shown to be independent of $\mathbf{k}$ and $\omega_{n}$ for $\mathbf{k}$ close to Fermi momentum. To evaluate the integrals in (8), such as $\int_{-\infty}^{+\infty} d \varepsilon \rho(\varepsilon)\left(\omega_{n}^{2}+E_{N}^{(j, v)}(k, B)^{2}\right)^{-1}$, we assume that $\rho(\varepsilon)=$ $\rho_{0} \delta\left(\varepsilon-E_{N}^{(j, v)}(k, B)\right)$, where we take a broad band-width, say, $(10 t) \sim 2.5 \mathrm{eV}$ which gives $\rho_{0} \sim 0.4(\mathrm{eV})^{-1}$. We thus obtain

$$
\begin{gathered}
G_{N}\left(\mathbf{k}, \omega_{n}\right) \approx-\rho_{0}\left(i \omega_{n}\right)\left(\frac{\pi}{\left|\omega_{n}\right|}\right), \\
\Sigma_{0}^{(1)}\left(\mathbf{k}, \omega_{n}\right)=-N_{j} \rho_{0}\left(i \omega_{n}\right) \sum_{k^{\prime}}\left|V\left(\mathbf{k}-\mathbf{k}^{\prime}\right)\right|^{2}\left(\frac{\pi}{\left|\omega_{n}\right|}\right) .
\end{gathered}
$$

We may write the right-hand side of (9b) as $\left[-i \omega_{n} /\left(2\left|\omega_{n}\right| \tau_{k}\right)\right]$, where $\left(1 / \tau_{k}\right)=2 \pi N_{j} \rho_{0} \Sigma_{k^{\prime}}\left|V\left(\mathbf{k}-\mathbf{k}^{\prime}\right)\right|^{2}$. Note that $\tau_{k}$, which corresponds to quasiparticle lifetime (QPLT), is expressed in reciprocal energy units. Upon using Dyson's equation, the full propagator may be written as $G_{N}^{\text {(Full) }}\left(\mathbf{k}, \omega_{n}\right) \approx \sum_{\nu= \pm 1}\{D / E\}$, where

$$
\begin{gathered}
D=\left[V_{k, N}^{(U, v) 2}\left(i \omega_{n}-E_{N}^{(L, v)}\right)+V_{k, N}^{(L, v) 2}\left(i \omega_{n}-E_{N}^{(U, v)}\right)\right], \\
E=\left(i \omega_{n}\right)^{2}-\left(i \omega_{n}\right)\left(E_{N}^{(U, v)}+E_{N}^{(L, v)}-\left(\frac{i}{2 \tau_{k}}\right)+\Sigma_{e}\right) \\
+\left\{E_{N}^{(U, v)} E_{N}^{(L, v)}+\left(-\left(\frac{i}{2 \tau_{k}}\right)+\Sigma_{e}\right)\right. \\
\left.\times\left(V_{k, N}^{(U, v) 2} E_{N}^{(L, v)}+V_{k, N}^{(L, v) 2} E_{N}^{(U, v)}\right)\right\} .
\end{gathered}
$$


We have dropped the argument part from the single-particle excitation spectra above for convenience. The roots of the equation $E=0$ are

$$
\begin{aligned}
i \omega_{n}= & \left\{\frac{\left(E_{N}^{(U, v)}+E_{N}^{(L, v)}-\left(i / 2 \tau_{k}\right)+\Sigma_{e}\right)}{2}\right\} \\
& \pm\left(\frac{R_{k, N}^{(v)} 1 / 2}{2}\right)\left(\cos \left(\frac{\theta_{k, N}^{(v)}}{2}\right)-i \sin \left(\frac{\theta_{k, N}^{(v)}}{2}\right)\right), \\
R_{k, N}^{(v)}= & {\left[E_{1}^{(v) 2}+E_{2}^{(\nu) 2}\right]^{1 / 2}, \quad \tan \left(\theta_{k, N}^{(v)}\right)=\frac{E_{2}^{(\nu)}}{E_{1}^{(\nu)}}, } \\
E_{1}^{(\nu)}= & \left(E_{N}^{(U, v)}-E_{N}^{(L, v)}\right)^{2}+\Sigma_{e}^{2} \\
& -\left(\frac{1}{4 \tau_{k}^{2}}\right)+2\left(E_{N}^{(U, v)}+E_{N}^{(L, v)}\right) \Sigma_{e} \\
& -4 \Sigma_{e}\left(V_{k, N}^{(U, v) 2} E_{N}^{(L, v)}+V_{k, N}^{(L, v) 2} E_{N}^{(U, v)}\right), \\
E_{2}^{(\nu)}= & \left\{\frac{\left(E_{N}^{(U, v)}+E_{N}^{(L, v)}+\Sigma_{e}\right)}{\tau_{k}}\right\} \\
& -\left(\frac{2}{\tau_{k}}\right)\left(V_{k, N}^{(U, v) 2} E_{N}^{(L, v)}+V_{k, N}^{(L, v) 2} E_{N}^{(U, v)}\right) .
\end{aligned}
$$

It follows that the denominator $E$ of $G_{N}^{\text {Full }}\left(\mathbf{k}, \omega_{\mathrm{n}}\right)$ may be written as the product of two factors $\left(i \omega_{n}-\left(\alpha_{v, N}^{(+)}+\right.\right.$ $\left.\left.i \beta_{v, N}^{(+)}\right)\right)\left(i \omega_{n}-\left(\alpha_{v, N}^{(-)}+i \beta_{\gamma, N}^{(-)}\right)\right)$, where

$$
\begin{aligned}
& \alpha_{\nu, N}^{(j= \pm)}=\left\{\frac{\left(E_{N}^{(U, v)}+E_{N}^{(L, v)}+\Sigma_{e}\right)}{2}\right\} \pm\left(\frac{R_{k, N}^{(\nu)} / 2}{2}\right) \cos \left(\frac{\theta_{k, N}^{(v)}}{2}\right), \\
& \beta_{\nu, N}^{(j= \pm)}=-\left\{\left(\frac{1}{4 \tau_{k}}\right) \pm\left(\frac{R_{k, N}^{(\nu)} 1 / 2}{2}\right) \sin \left(\frac{\theta_{k, N}^{(v)}}{2}\right)\right\} .
\end{aligned}
$$

In view of $(12), G^{\text {Full }}{ }_{N}\left(\mathbf{k}, \omega_{\mathrm{n}}\right) \approx \sum_{\nu= \pm 1}\{D / E\}$ may be written as

$$
\begin{aligned}
G_{N}^{(\text {Full })}\left(\mathbf{k}, \omega_{n}\right)= & \sum_{\nu= \pm 1} V_{\text {ren, },, N}^{(+, v) 2}\left[i \omega_{n}-\dot{\varepsilon}_{r}^{(+, v)} N_{N}+i\left(\frac{1}{4 \tau_{k, N}^{(+, \nu)}}\right)\right]^{-1} \\
& +V_{\text {ren, },, N}^{(-, \nu) 2}\left[i \omega_{n}-\dot{\varepsilon}_{r}^{(-, v)}+i\left(\frac{1}{4 \tau_{k, \alpha, N}^{(-, v)}}\right)\right]^{-1},
\end{aligned}
$$

where the superscript $j=( \pm)$,

$$
\begin{gathered}
\dot{\varepsilon}_{r}^{(j, v)}{ }_{N}=\alpha_{\nu, N}^{(j)}, \\
\left(1 / \tau_{k, N}^{(j, v)}\right)=\left\{\left(1 / \tau_{k}\right) \pm\left(R_{k, N}^{(\nu)} 1 / 2\right) \sin \left(\theta_{k, N}^{(\nu)} / 2\right)\right\}, \\
V_{\text {ren, }, k, N}^{(j, v) 2}=\left(\frac{1}{4}\right)\left(1 \pm \delta_{k, N}\right), \quad \delta_{k, N}=\left(\frac{\delta_{k, N}^{(1)}}{\delta_{k, N}^{(2)}}\right), \\
\delta_{k, \alpha, N}^{(1)}=\left[\left(\alpha_{v, N}^{(+)}+i \beta_{\nu, N}^{(+)}\right)+\left(\alpha_{\nu, N}^{(-)}+i \beta_{\nu, N}^{(-)}\right)\right. \\
\left.-2\left(V_{k, N}^{(+, v) 2} E_{N}^{(-, v)}+V_{k, N}^{(-, v) 2} E_{N}^{(+, v)}\right)\right], \\
\delta_{k, N}^{(2)}=\left[\left(\alpha_{v, N}^{(+)}+i \beta_{v, N}^{(+)}\right)-\left(\alpha_{v, N}^{(-)}+i \beta_{\nu, N}^{(-)}\right)\right] .
\end{gathered}
$$

The renormalized Bogoliubov coherence factors $V_{\mathrm{ren}, k, N}^{(j, v) 2}$ turn out to be complex quantities.

We have calculated explicitly the propagators $G_{N}^{\text {Full }}\left(\mathbf{k}, \omega_{n}\right)$ above with the inclusion of impurity scattering. The corresponding retarded Green function $G_{N}^{(R)}(\mathbf{k}, t)$, in units such that $\hbar=1$, is given by $G_{N}^{(R)}(\mathbf{k}, t)=\int_{-\infty}^{+\infty}(d \omega / 2 \pi) \exp (-i \omega t) G_{N}^{(\text {Full })}(\mathbf{k}, \omega)$, where in the upper and lower half-plane, respectively,

$$
\begin{aligned}
& G_{N}^{(\text {Full })}\left(\mathbf{k}, \omega_{n}\right) \\
& =\sum_{j=( \pm), v= \pm 1} V_{\text {ren }, k, N}^{(j, v)}\left[i \omega_{n}-\hat{\varepsilon}_{r}^{(j, v)}{ }_{N}+\left(\frac{i}{4 \tau_{k, N}^{(j, v)}}\right)\right]^{-1}, \\
& G_{N}^{(\text {Full })}\left(\mathbf{k}, \omega_{n}\right) \\
& =\sum_{j=( \pm), v= \pm 1} V_{\text {ren }, k, N}^{(j, v) 2}\left[i \omega_{n}-\hat{\varepsilon}_{r}^{(j, v)}{ }_{N}-\left(\frac{i}{4 \tau_{k, N}^{(j, v)}}\right)\right]^{-1} .
\end{aligned}
$$

Thus $G_{N}^{(R)}\left(\mathbf{k}, \omega^{\prime}\right)=\int_{-\infty}^{+\infty} d t \exp \left(i \omega^{\prime} t\right) G_{N}^{(R)}(\mathbf{k}, t)$ is given by $(16)$ with $\omega$ real. We obtain

$$
\begin{aligned}
& G_{N}^{(R)}(\mathbf{k}, t) \\
& =\sum_{j=( \pm), v= \pm 1} V_{\mathrm{ren}, k, N}^{(j, v) 2} i \exp \left(-i \hat{\varepsilon}_{r}^{(j, v)}{ }_{N}(\mathbf{k}) t-\left(\frac{t}{4 \tau_{k, N}^{(j, v)}}\right)\right) \theta(t),
\end{aligned}
$$

where the unit step function $\theta(t)=\int_{-\infty}^{+\infty}(i d \omega / 2 \pi)\{\exp (-i \omega t) /$ $\left.\left(\omega+i 0^{+}\right)\right\}$. The electronic excitations in cuprates are thus demonstrably Bogoliubov quasiparticles in the pseudogap phase with finite lifetime for the states of definite momentum 
due to the impurity scattering. Using the integral representation of $\theta(t)$ above, it is not difficult to show that

$$
\begin{aligned}
G_{N}^{(R)}\left(\mathbf{k}, \omega^{\prime}\right)= & \int_{-\infty}^{+\infty} d t \exp \left(i \omega^{\prime} t\right) G_{N}^{(R)}(\mathbf{k}, t) \\
= & \sum_{j=( \pm), v= \pm 1} V_{\mathrm{ren}, k, N}^{(j, v) 2}\left[\omega^{\prime}-\hat{\varepsilon}_{r}^{(j, v)}{ }_{N}+\left(\frac{i}{4 \tau_{k, N}^{(j, v)}}\right)\right] \\
& \times\left[\left(\omega^{\prime}-\hat{\varepsilon}_{r}^{(j, v)}{ }_{N}\right)^{2}+\left(\frac{1}{4 \tau_{k, N}^{(j, v)}}\right)^{2}\right]^{-1} .
\end{aligned}
$$

As the renormalized Bogoliubov coherence factors $V_{\text {ren, } k, N}^{(j, v) 2}$ are found to be complex, the dimensionless density of states $\rho_{N}(\mathbf{k}, \omega) \equiv\left(-1 / 2 \pi^{2} \rho_{0}\right) \operatorname{Im} G_{N}^{(R)}(\mathbf{k}, \omega)$ comprises of two parts: $\rho_{N}(\mathbf{k}, \omega)=\rho_{N}^{\prime}(\mathbf{k}, \omega)+\rho_{N}^{\prime \prime}(\mathbf{k}, \omega)$, where

$$
\begin{aligned}
\rho_{N}^{\prime}(\mathbf{k}, \omega)= & \left(\frac{1}{2 \pi^{2} \rho_{0}}\right) \sum_{j=( \pm), v= \pm 1}\left(\operatorname{Re} V_{\mathrm{ren}, k, N}^{(j, v) 2}\right) \gamma_{k, N}^{(j, v)} \\
& \times\left[\left(\omega-\hat{\varepsilon}_{r}^{(j, v)}{ }_{N}(\mathbf{k})\right)^{2}+\gamma_{k, N}^{(j, v) 2}\right]^{-1}, \\
\rho_{\alpha, N}^{\prime \prime}(\mathbf{k}, \omega)= & \left(-\frac{1}{\pi \rho_{0}}\right) \sum_{j=( \pm), v= \pm 1}\left(\operatorname{Im} V_{\mathrm{ren}, k, N}^{(j, v) 2}\right) \\
& \times\left(\omega-\hat{\varepsilon}_{r}^{(j, v)}{ }_{N}(\mathbf{k})\right) \\
& \times\left[\left(\omega-\hat{\varepsilon}_{r}^{(j, v)}{ }_{N}(\mathbf{k})\right)^{2}+\gamma_{k, N}^{(j, v) 2}\right]^{-1}, \\
\operatorname{Re} V_{\mathrm{ren}, k, N}^{(j, v) 2}= & \left(\frac{1}{4}\right)\left(1 \pm \operatorname{Re} \delta_{k, \alpha, N}\right), \\
\operatorname{Im} V_{\mathrm{ren}, k, N}^{(j, v) 2}= & \left(\frac{1}{4}\right)\left(1 \pm \operatorname{Im} \delta_{k, \alpha, N}\right),
\end{aligned}
$$

and $\gamma_{k, N}^{(j, v)}=\tau_{k, N}^{(j, v)-1} / 4$ (the level-broadening factors). In order to determine the Fermi energy density of states (DOS) $\rho_{N \text {,Fermi }}(\mathbf{k})$, since we shall put $\omega=\mu$ in (20) and (21), it is clear that $\rho_{N, \text { Fermi }}(\mathbf{k})=\sum_{j=( \pm), \nu= \pm 1} \rho_{N, \text { Fermi }}^{(j, v)}(\mathbf{k})$, where

$$
\begin{aligned}
\rho_{N, \mathrm{Fermi}}^{(j, v)}(\mathbf{k})= & \left(\frac{1}{2 \pi^{2} \rho_{0}}\right)\left(\operatorname{Re} V_{\mathrm{ren}, k, N}^{(j, v) 2}\right) \gamma_{k, N}^{(j, v)} \\
& \times\left[\left(\mu-\hat{\mathcal{\varepsilon}}_{r}^{(j, \nu)}{ }_{N}(\mathbf{k})\right)^{2}+\gamma_{k, N}^{(j, v) 2}\right]^{-1} .
\end{aligned}
$$

Equation (21) does not contribute here as the branches of the Fermi surface are given by $\left(\hat{\varepsilon}_{r}^{(j, v)}{ }_{N}(\mathbf{k})-\mu\right)=0$. However, for $\omega \neq \mu$, definitely this equation will contribute towards the DOS. The chemical potential $\mu$, according to the Luttinger rule, is given by the equation

$$
\begin{aligned}
(1+p)= & \int d(\mathbf{k} a) \sum_{j, v, N} \rho_{N, \mathrm{Fermi}}^{(j, v)}(\mathbf{k}) \\
& \times\left(\exp \left(\beta\left(\dot{\varepsilon}_{r}^{(j, v)}{ }_{N}(\mathbf{k}, B)-\mu\right)\right)+1\right)^{-1}
\end{aligned}
$$

where $p$ is the doping level, $\int d(\mathbf{k} a) \rightarrow \int_{-\pi}^{+\pi}\left(d\left(k_{x} a\right) /\right.$ $2 \pi \int_{-\pi}^{+\pi}\left(d\left(k_{y} a\right) / 2 \pi\right.$, and $\beta=\left(k_{B} T\right)^{-1}$. It must be mentioned that $\rho_{N, \text { Fermi }}(\mathbf{k}, B=0)$ roughly corresponds to the so-called "maximal intensity surface" [24, 25] of the ARPES studies, provided the momentum dependence of the level broadening factors is ignored.

We now model $V\left(\left|\mathbf{k}-\mathbf{k}^{\prime}\right|\right)$ by a screened exponential falloff of the form $V\left(\left|\mathbf{k}-\mathbf{k}^{\prime}\right|\right)=\left[\left|v_{0}\right|^{2} \kappa^{2} /\left\{\left|\mathbf{k}-\mathbf{k}^{\prime}\right|^{2}+\kappa^{2}\right\}\right]^{1 / 2}$ to consider the effect of the in-plane impurities, where $\kappa^{-1}$ characterizes the range of the impurity potential. The limit $\kappa \gg\left|\mathbf{k}-\mathbf{k}^{\prime}\right|$, which corresponds to a point-like isotropic scattering potential characterizing the in-plane impurities, will only be considered here for simplicity. At this stage, assuming low concentration of impurities, one may include the contributions of all such diagrams which involve only one impurity vertex. This gives the equation to determine the total self-energy $\Sigma_{N}\left(\mathbf{k}, \omega_{n}\right)$ :

$$
\Sigma_{N}\left(\mathbf{k}, \omega_{n}\right)=N_{j} \sum_{q} V(\mathbf{q}) G_{N}\left(\mathbf{k}-\mathbf{q}, \omega_{n}\right) \Gamma_{N}\left(\mathbf{k}, \mathbf{q}, \omega_{n}\right),
$$

where the Lippmann-Schwinger equation to determine $\Gamma_{N}\left(\mathbf{k}, \mathbf{q}, \omega_{n}\right)$ is

$$
\begin{aligned}
\Gamma_{N}\left(\mathbf{k}, \mathbf{q}, \omega_{n}\right)= & V(-\mathbf{q})+\sum_{q^{\prime}} V\left(\mathbf{q}^{\prime}-\mathbf{q}\right) G_{N}\left(\mathbf{k}-\mathbf{q}^{\prime}, \omega_{n}\right) \\
& \times \Gamma_{N}\left(\mathbf{k}, \mathbf{q}^{\prime}, \omega_{n}\right) .
\end{aligned}
$$

This is the t-matrix approximation.Upon using the optical theorem for the t-matrix [32], one may write

$$
\Sigma_{N}\left(\mathbf{k}, \omega_{n}\right)=i \operatorname{Im} \Gamma_{N}\left(k, k, \omega_{n}\right)=-\frac{i \omega_{n}}{\left(2\left|\omega_{n}\right| \hat{\Gamma}_{k, N}\right)},
$$

where $\hat{\Gamma}_{k, N}^{-1}=2 \pi N_{j} \rho_{0} \sum_{k^{\prime}}\left|\Gamma_{N}\left(\mathbf{k}, \mathbf{k}^{\prime}\right)\right|^{2}$. Thus the effect of the inclusion of contribution of all the above mentioned diagrams is to replace the Born approximation for scattering by the exact scattering cross-section for a single impurity, that is, $\tau_{k}^{-1} \rightarrow \hat{\Gamma}_{k}^{-1}$. Since $G_{N}\left(\mathbf{k}, \omega_{n}\right)$ and $V(\mathbf{q})$ are known, using (24), (25), and (26), one can determine $\Gamma_{k, N}^{-1}$ in terms of $V(\mathbf{k})$. In the limit $\kappa \gg\left|\mathbf{k}-\mathbf{k}^{\prime}\right|$, the disorder potential $V(|\mathbf{q}|) \approx\left|v_{0}\right|$, and, therefore, from the latter we obtain

$$
\Gamma_{N}\left(\mathbf{k}, \omega_{n}\right) \approx \frac{\left|v_{0}\right|}{\left(1-\left|v_{0}\right| G_{\alpha, N}\left(\mathbf{k}, \omega_{n}\right)\right)} .
$$

In view of $(9 a)$, we find that

$$
\operatorname{Im} \Gamma_{N}\left(\mathbf{k}, \omega_{n}\right) \approx \frac{-\rho_{0} \pi\left|v_{0}\right|^{2}}{\left(1+\rho_{0}^{2} \pi^{2}\left|v_{0}\right|^{2}\right)}
$$

From (27) we now find that $\Gamma_{k, N}^{-1}$, in the first approximation, is given by $\left[2 \rho_{0} \pi\left|v_{0}\right|^{2} /\left(1+\rho_{0}^{2} \pi^{2}\left|v_{0}\right|^{2}\right)\right]$. We take a moderate disorder potential $\left|v_{0}\right|=0.25 \mathrm{eV}$. This gives $\gamma_{\mathbf{k}, N}=\hat{\Gamma}_{k, N}^{-1} / 4 \approx$ $0.0357 \mathrm{eV}$. We next take a stronger disorder potential $\left|v_{0}\right|=$ $1 \mathrm{eV}$. This gives $\gamma_{\mathbf{k}, N}=\dot{\Gamma}_{k, N}^{-1} / 4 \approx 0.2436 \mathrm{eV}$. We note 
that, even though $\Gamma_{k, N}$ is found to be $\mathbf{k}$-independent in the first approximation, the term $\pm 4 R_{k, N}^{1 / 2} \sin \left(\theta_{k, N} / 2\right)$ in (15) will ensure that $\tau_{k, N}^{(j, v)}$ are momentum dependent and different for the upper and lower branches. It may be mentioned in passing that the total DOS $\rho_{N, \text { Fermi }}^{(j, v)}$ has been obtained by the summation of the density $\rho_{N, \text { Fermi }}^{(j, v)}(\mathbf{k})$ over the BZ and found to assume positive values although, as in Figures 2 and 3, $\rho_{N, \text { Fermi }}^{(j, v)}(\mathbf{k})$ becomes negative on some specific points of the BZ. For the $\mathbf{k}$-summation purpose, we first divide the BZ into finite number of rectangular patches. We next determine the numerical values corresponding to each of these patches of the momentum-dependent density $\rho_{N, \text { Fermi }}^{(j, v)}(\mathbf{k})$ and sum these values. We have generated these values through the surface plots above. With the inputs obtained in this section, we embark on a calculation of the specific heat in the next section.

\section{Magneto-Quantum Oscillations in Specific Heat}

Following the Kadanoff-Baym approach [33], the thermodynamic potential may be given by the expression

$$
\begin{aligned}
& \Omega(T, B, \mu) \\
& =\Omega_{0}(B)-2\left(\beta N_{s}\right)^{-1} \times \sum_{j, k, v, N} \ln \cosh \left(\frac{\beta\left(\hat{\varepsilon}_{r}^{(j, v)}{ }_{N}(\mathbf{k}, B)-\mu\right)}{2}\right),
\end{aligned}
$$

where $\Omega_{0}(B)=N_{s}^{-1} \sum_{j, k, v, N}\left(\left(\dot{\varepsilon}_{r}^{(j, v)}{ }_{N}(\mathbf{k}, B)-\mu\right)\right.$ and $\beta=$ $\left(k_{B} T\right)^{-1}$. The dimensionless entropy per unit cell is given by $S=\beta^{2}(\partial \Omega / \partial \beta)$ while the electronic specific heat, for the pseudogapped (PG) phase $\left(T<T^{*}\right)$, is $C_{\mathrm{el}}=-\beta(\partial S / \partial \beta)$. We obtain

$$
\begin{aligned}
C_{\mathrm{el}} \approx & 2 k_{B} N_{s}^{-1} \sum_{k, j, v, N}\left(\beta\left(\dot{\varepsilon}_{r}^{(j, v)}{ }_{N}(\mathbf{k}, B)--\mu\right)\right)^{2} \\
& \times \exp \left(\beta\left(\hat{\varepsilon}_{r}^{(j, v)}{ }_{N}(\mathbf{k}, B)-\mu\right)\right) \\
& \times\left(\exp \left(\beta\left(\dot{\varepsilon}_{r}^{(j, v)}{ }_{N}(\mathbf{k}, B)-\mu\right)\right)+1\right)^{-2} .
\end{aligned}
$$

We have ignored the temperature dependence of the chemical potential above. Now strictly speaking, for the magnetic field-dependent phenomena, whenever we need to replace the sum over physical momenta $\mathbf{k}$ by an integral over a region of $\mathbf{k}$-space, the Berry-phase-corrected result is to be used. It is known $[1,2]$ that for some isolated points in $\mathbf{k}$-space, the correction is much larger than those corresponding to the other points for a magnetic field $B$ even of order 1 Tesla. In what follows, we, however, ignore this correction. Upon using (23) in (31) we find that the electronic specific heat at a given doping level is given by $C_{\mathrm{el}} \approx \gamma(B) T$, where the specific heat coefficient for $B \neq 0$ may be expressed as

$$
\begin{aligned}
\gamma(B) \approx & \left(\frac{k_{B}^{2}}{\pi^{2}}\right) \sum_{j, v, N} \int d(\mathbf{k} a) Q_{1}(B, \mathbf{k}) \times Q_{2}(B, \mathbf{k}), \\
Q_{1}(B, \mathbf{k})= & \left(\frac{\gamma_{\mathbf{k}, N}^{(j, v)}}{\rho_{0}}\right) \times\left[\left(\mu-\hat{\varepsilon}_{r}^{(j, v)}{ }_{N}(\mathbf{k})\right)^{2}+\gamma_{\mathbf{k}, N}^{(j, v)}\right]^{-1}, \\
Q_{2}(B, \mathbf{k})= & \left(\operatorname{Re} V_{\mathrm{ren}, k, N}^{(j, v)}\right) \times \beta \times\left(\beta\left(\hat{\varepsilon}_{r}^{(j, v)}{ }_{N}(\mathbf{k}, B)-\mu\right)\right)^{2} \\
& \times \exp \left(\beta\left(\hat{\varepsilon}_{r}^{(j, v)}{ }_{N}(\mathbf{k}, B)-\mu\right)\right) \\
& \times\left(\exp \left(\beta\left(\hat{\varepsilon}_{r}^{(j, v)}{ }_{N}(\mathbf{k}, B)-\mu\right)\right)+1\right)^{-2} .
\end{aligned}
$$

The quantity $Q_{2}(B, \mathbf{k})$ is given by the expression

$$
Q_{2}(B, \mathbf{k})=\int_{-\infty}^{+\infty} d x I_{k, N}^{(j, v)}(x, B)\left\{\frac{x^{2} e^{x}}{\left(e^{x}+1\right)^{2}}\right\},
$$

where

$$
\begin{aligned}
I_{k, N}^{(j, v)}(x, B)= & \left(\hbar \omega_{c}\right)^{-1}\left(\operatorname{Re} V_{\mathrm{ren}, k, N}^{(j, v) 2}\right) \\
& \times \delta\left(\left(\frac{x}{\beta \hbar \omega_{c}}\right)-\left(\hbar \omega_{c}\right)^{-1}\left(\dot{\varepsilon}_{r}^{(j, v)}{ }_{N}(\mathbf{k}, B)-\mu\right)\right) .
\end{aligned}
$$

The delta functions can be expanded in cosine Fourier series: $\delta(x-a)=(2 \pi)^{-1}+\pi^{-1} \sum_{m=1}^{\infty} \cos [m(x-a)]$. Upon doing so, we find that the nonoscillatory part of the specific heat, with the linear dependence on $T$, is

$$
C_{\text {nonoscl }} \sim\left(\frac{k_{B}^{2} T}{3 \pi \hbar \omega_{c}}\right) \sum_{j, v, N} \int d(\mathbf{k} a) Q_{1}(B, \mathbf{k}) \times\left(\operatorname{Re} V_{\mathrm{ren}, k, N}^{(j, v) 2}\right) .
$$

The oscillatory part may be expressed as

$$
\begin{aligned}
C_{\text {oscll }}= & \left(k_{B}^{2} \frac{T}{\pi^{3}} \hbar \omega_{c}\right) \sum_{j, \nu, N} \int d(\mathbf{k} a) Q_{1}(B, \mathbf{k}) \times\left(\operatorname{Re} V_{\mathrm{ren}, k, N}^{(j, v) 2}\right) \\
& \times \int_{-\infty}^{+\infty} d x \sum_{m=1}^{\infty} \cos \left(\frac{m x}{\beta \hbar \omega_{c}}\right)\left\{\frac{x^{2} e^{x}}{\left(e^{x}+1\right)^{2}}\right\} \\
& \times \cos \left\{m\left(\hbar \omega_{c}\right)^{-1}\left(\dot{\varepsilon}_{r}^{(j, v)}{ }_{N}(\mathbf{k}, B)-\mu\right)\right\} .
\end{aligned}
$$

Equation (36) prima facie indicates that the origin of the approximate $(1 / B)$-oscillations in the electronic specific heat of the underdoped YBCO is the upper and the lower branches of the excitation spectrum. The appearance of the Dingle factors in (36), due to the scattering by impurities, is ensured by the Lorentzian $Q_{1}(B, \mathbf{k})$. In this Lorentzian, for the moderate disorder potential $\left|v_{0}\right|=0.25 \mathrm{eV}$, the level broadening factor (LBF) is $0.0357 \mathrm{eV}$ while, for the stronger disorder potential $\left|v_{0}\right|=1 \mathrm{eV}$, the same is $0.2436 \mathrm{eV}$. 


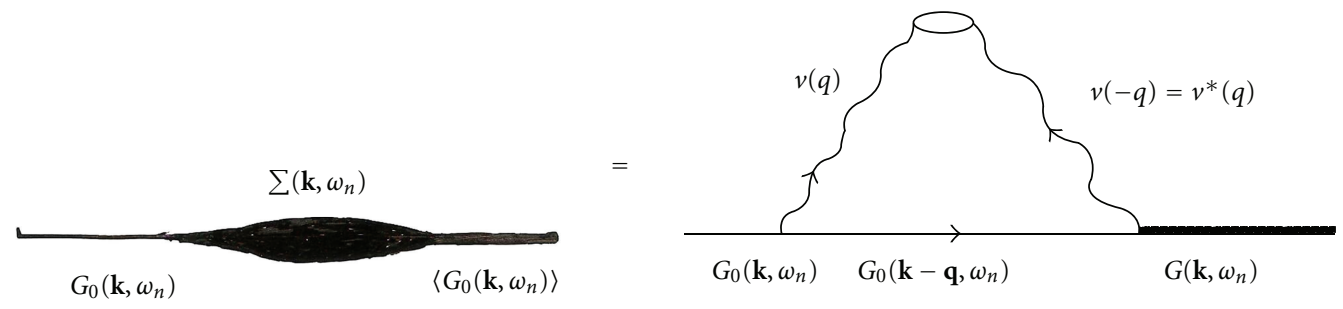

(a)

$v\left(q_{1}\right)$

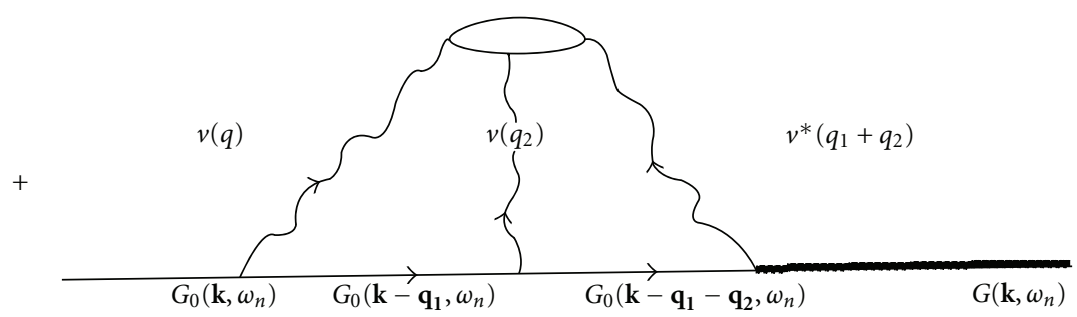

(b)

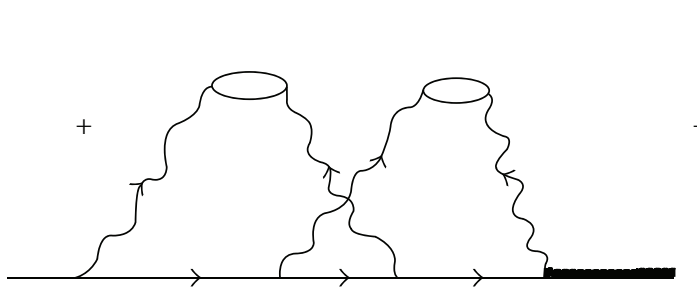

(c)

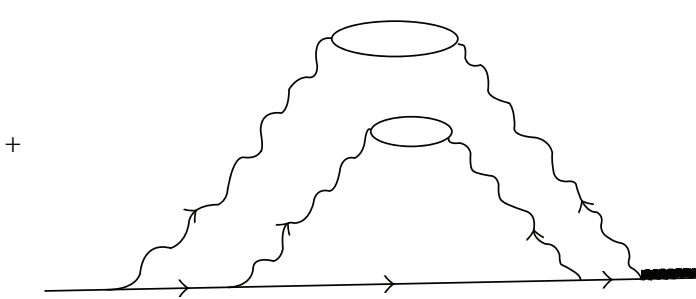

(d)

Figure 1: A few diagrams contributing to the self-energy. The wiggly lines carry momentum but no energy. The total momentum entering each impurity vertex, depicted by a slim ellipse, is zero. We have assumed that impurities are alike and distributed randomly. Whereas Figures 1(a) and 1(b) correspond to one impurity vertex, Figures 1(c) and 1(d) correspond to a product of four impurity potentials with nonzero averages. These are the cases where two impurities each gives rise to two potentials. Thus the figures involve the interference of the scattering by more than one impurity. We have assumed low concentration of impurities and therefore these figures yield smaller contributions compared to those corresponding to Figures 1 (a), 1(b), and the other diagrams of the same class involving only one impurity vertex.

In an effort to explain the possible quantum oscillations in the specific heat, for the anisotropy parameter $\dot{\varepsilon}=0$ with LL index $N=0$ and 1 , we introduce the two quantities $\widetilde{\widehat{E}}_{a n, N}$ and $\widetilde{\widehat{E}}_{n, N}$, where

$$
\begin{aligned}
&\left.\dot{\varepsilon}_{r}^{( \pm, v)}(\mathbf{k}, B)\right|_{\mathbf{k}=[( \pm \pi, 0),(0, \pm \pi)]} \equiv \widetilde{E}_{a n, N}(B), \\
&\left.\dot{\varepsilon}_{r}^{( \pm, v)}(\mathbf{k}, B)\right|_{\mathbf{k}=( \pm \pi / 2, \pm \pi / 2)} \equiv \widetilde{\hat{E}}_{n, N}(B),
\end{aligned}
$$

corresponding to the anti-nodal and the nodal excitations respectively, and calculate these quantities for the disorder potential $\left|v_{0}\right|=0.25 \mathrm{eV}$ and $1 \mathrm{eV}$. Each of the quantities $\widetilde{\widehat{E}}_{a n, N}(B)$ and $\widetilde{\widehat{E}}_{n, N}(B)$ will have 8 values corresponding to the superscripts $( \pm, v)$ and the subscript $N$. Since we take
$N=0$ and 1 , the $4 \times 4=16$ possible values are displayed in Table 1 for $B=0$; likewise, $8 \times 4=32$ possible values are in Table 2 for $B=45 \mathrm{~T}$. From Tables 1 and 2 (see also table legends below) we clearly notice that as the magnetic field $(B)$ is increased, provided one is in the weak disorder regime $\left(\left|v_{0}\right|=0.25 \mathrm{eV}\right)$, the Landau states move to a higher energy, ultimately rising above the Fermi level. They are thereby emptied, and the excess fermions find a place in the next lower Landau level (LL). During the crossing of an LL, its occupation by fermions is halted and then reduced. The specific heat consequently decreases slightly. As the excess fermions get accommodated in the next lower LL, the specific heat rises again. Thus the oscillations in the fermion density in the vicinity of Fermi energy manifest themselves as oscillations in the specific heat. These events 


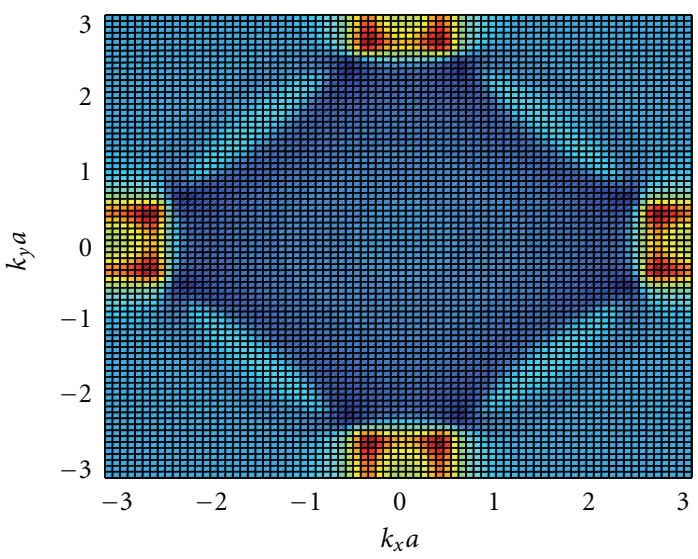

(a)

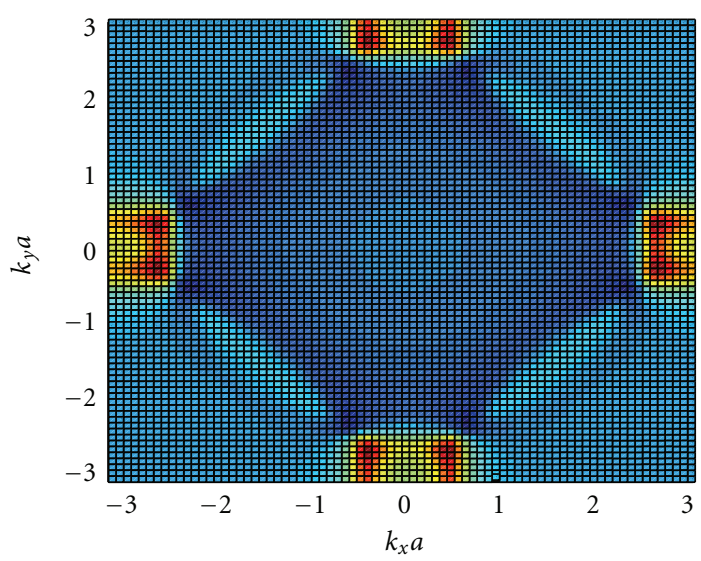

(c)

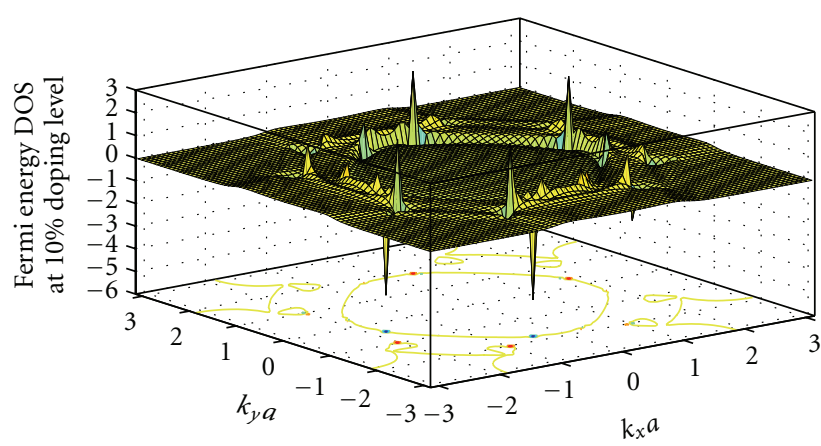

(b)

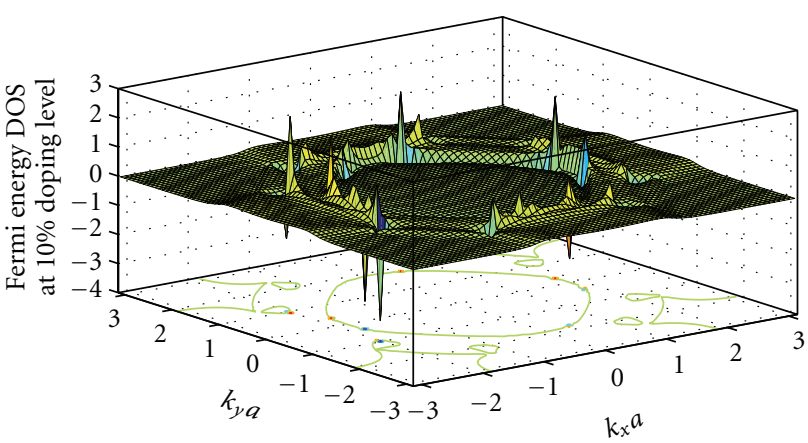

(d)

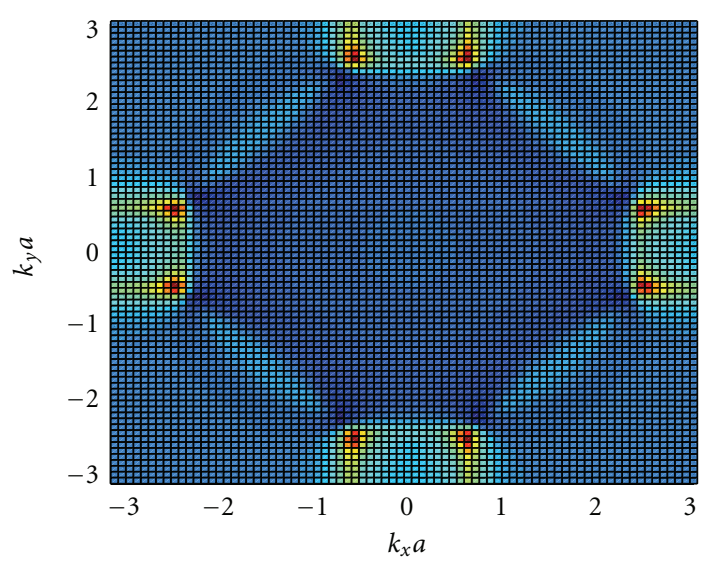

(e)

Figure 2: The contour/3D plots of the Fermi energy DOS on the Brillouin zone (BZ) at 10\% hole doping, $\left|t^{\prime} / t\right|=0.4$ (except (e)) and $B=0$. (a) The anisotropy parameter $\dot{\varepsilon}=0$ and the level broadening factor $\gamma=0.0357 \mathrm{eV}$. (b) $\dot{\varepsilon}=0$ and $\gamma=0.2436 \mathrm{eV}$. (c) $\dot{\varepsilon}=0.05$ and $\gamma=0.0357 \mathrm{eV}$. (d) $\dot{\varepsilon}=0.05$ and $\gamma=0.2436 \mathrm{eV}$. (e) $\dot{\varepsilon}=0.05, \gamma=0.0357 \mathrm{eV}$, and $\left|t^{\prime} / t\right|=0.5$. The scale of the plots in Figures 2 (a), 2 (c) and $2(\mathrm{e})$ is from -0.2 to 1.2 . In the weak disorder regime we obtain prominent electron pockets and barely visible hole-like patches. In the strong disorder regime the scenario does not change radically. We have assumed $\Sigma_{e}=0.05 \mathrm{eV}$ above. The comparison of figures 2 (a) with 2(c) (and figures 2(b) with 2(d)) indicates the slight change (Pomeranchuk distortion) in the topology of the Fermi surface due to $\dot{\varepsilon} \neq 0$; electron pockets around $(0, \pm \pi)$ widen slightly compared to those around $( \pm \pi, 0)$. (e) The figure indicates that the higher value of the ratio $\left|t^{\prime} / t\right|$ may lead to the disappearance of the electron pockets. 


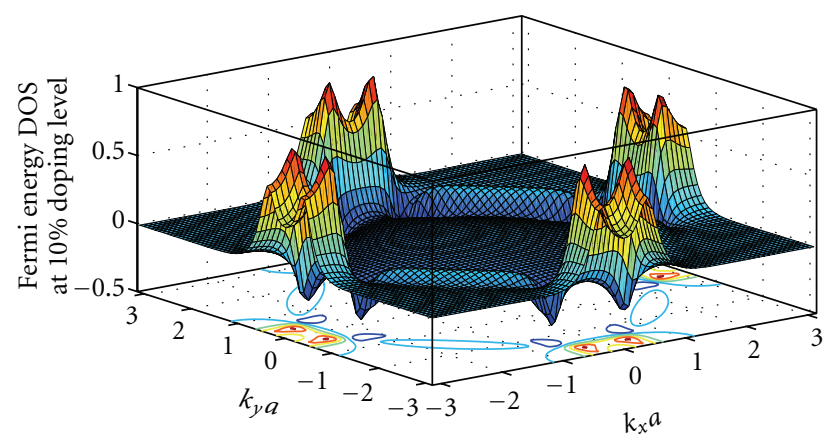

(a)

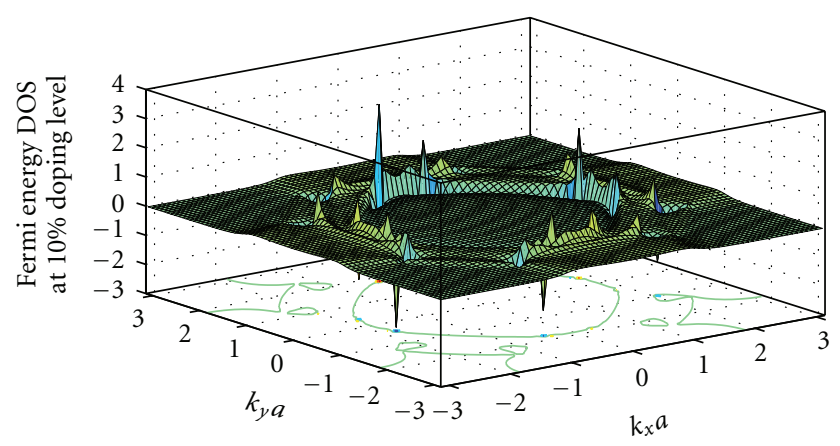

(b)

Figure 3: The 3D plots of the Fermi energy DOS on the Brillouin zone (BZ) at $10 \%$ hole doping and $B=45$ Tesla. (a) The anisotropy parameter $\dot{\varepsilon}=0$ and the level broadening factor $\gamma=0.0357 \mathrm{eV}$. (b) $\dot{\varepsilon}=0$ and $\gamma=0.2436 \mathrm{eV}$. The features for $B=45 \mathrm{~T}$ are by and large the same as in Figure 2.

take place for $17 \mathrm{~T} \leq B \leq 53 \mathrm{~T}$ (see the table legends). The similar oscillations in the electrical conductivity (Shubnikovde Haas oscillations (SdHOs)) are already reported [13-16]. As we notice above, in YBCO, these oscillations have their origin at the electron pockets in the Fermi surface located around the anti-nodal points provided one is in the weak disorder regime $\left(\left|v_{0}\right|=0.25 \mathrm{eV}\right)$. For the strong disorder potential $\left(\left|v_{0}\right|=1 \mathrm{eV}\right)$, the energy values $\widetilde{\hat{E}}_{a n, N}(B=0)$ in Table 1 , less than $\mu=-0.27 \mathrm{eV}$, are too far below $\mu$ to be able to contribute to the oscillation process up to a reasonably high magnetic field $B$ of order $50 \mathrm{~T}$. The basic reason, as we infer, behind this magneto-oscillation phenomenon is the Landau quantization in the presence of a strong magnetic field $(B>17 \mathrm{~T})$ (In weak magnetic fields $(B<1 \mathrm{~T})$, i.e. in the linear response regime, one can neglect Landau quantization, since the existence of Landau levels (LLs) in small magnetic fields requires extremely clean and, possibly very large samples) [31] in a reasonably clean sample. The point to be noted here is that one cannot afford to have the total absence of disorder, for in that case the level broadening factors $\gamma$ will be zero in (23) for $\rho_{N, \text { Fermi }}^{(j, v)}(\mathbf{k})$. Thus, in any investigation on the quantum oscillations, including a recent noteworthy theoretical work [34] involving the Fermi arc picture, the inclusion of moderate disorder seems to be necessary.
TABLE 1: The values of the anti-nodal and the nodal excitations for $B=0$ for the disorder potential $\left|v_{0}\right|=0.25 \mathrm{eV}$ and $1 \mathrm{eV}$. All the values of $\widetilde{\hat{E}}_{n, N}(B=0)$ are above the value of $\mu=-0.27 \mathrm{eV}$. Therefore, the origin of the specific heat oscillations shown in (36) would not correspond to the nodal patches around $k=$ $( \pm \pi / 2, \pm \pi / 2)$ shown in Figure 2 .

\begin{tabular}{cccc}
\hline \multicolumn{2}{c}{$\left|v_{0}\right|=0.25 \mathrm{eV}$} & \multicolumn{2}{c}{$\left|v_{0}\right|=1 \mathrm{eV}$} \\
$\widehat{\widehat{E}}_{a n, N}(B=0)$ & $\widetilde{\widehat{E}}_{n, N}(B=0)$ & $\widetilde{\widehat{E}}_{a n, N}(B=0)$ & $\widetilde{\widehat{E}}_{n, N}(B=0)$ \\
in eV & in eV & in eV & in eV \\
\hline-0.2578 & 0.1639 & -0.0857 & 0.3389 \\
$-0.5130^{*}$ & 0.0249 & $-0.6871^{*}$ & -0.1501 \\
$-0.2724^{* *}$ & 0.1639 & -0.0982 & 0.3389 \\
$-0.5324^{*}$ & 0.0249 & $-0.7066^{*}$ & -0.1501 \\
\hline
\end{tabular}

* The energy values are less than $\mu=-0.27 \mathrm{eV}$.

$* *$ The energy value is the closest to $\mu=-0.27 \mathrm{eV}$.

TABLE 2: The values of the anti-nodal and the nodal excitations for $B=45 \mathrm{~T}$ for the disorder potential $\left|v_{0}\right|=0.25 \mathrm{eV}$ and $1 \mathrm{eV}$. The first four rows correspond to LL index $N=0$ while the next four to $N=1$. All the values of $\widetilde{\widehat{E}}_{n, N}(B=45 \mathrm{~T})$ are above the value of $\mu=-0.27 \mathrm{eV}$.

\begin{tabular}{cccc}
\hline \multicolumn{2}{c}{$\left|v_{0}\right|=0.25 \mathrm{eV}$} & \multicolumn{2}{c}{$\left|v_{0}\right|=1 \mathrm{eV}$} \\
$\widetilde{\widehat{E}}_{a n, N}(B=45 \mathrm{~T})$ & $\widetilde{\widehat{E}}_{n, N}(B=45 \mathrm{~T})$ & $\widetilde{\widehat{E}}_{a n, N}(B=45 \mathrm{~T})$ & $\widetilde{\widehat{E}}_{n, N}(B=45 \mathrm{~T})$ \\
in eV & in eV & in eV & in eV \\
\hline-0.2578 & 0.1695 & -0.0837 & 0.3418 \\
$-0.5098^{*}$ & 0.0245 & $-0.6839^{*}$ & -0.1478 \\
$-0.2703^{* *}$ & 0.1695 & -0.0962 & 0.3418 \\
$-0.5293^{*}$ & 0.0245 & $-0.7034^{*}$ & -0.1478 \\
-0.2538 & 0.1766 & -0.0797 & 0.3472 \\
$-0.5035^{*}$ & 0.0278 & $-0.6775^{*}$ & -0.1429 \\
$-0.2662^{* * *}$ & 0.1766 & -0.0921 & 0.3472 \\
$-0.5230^{*}$ & 0.0278 & $-0.6971^{*}$ & -0.1429 \\
\hline
\end{tabular}

* The energy values are less than $\mu=-0.27 \mathrm{eV}$.

${ }^{*} *$ This is the energy value (corresponding to LL index $N=0$ ) which increases to $-0.2700 \mathrm{eV}$ at $B=53 \mathrm{~T}$.

${ }^{* * *}$ This is the energy value (corresponding to $N=1$ ) which attains the value of $\mu=-0.2700 \mathrm{eV}$ at $B=17 \mathrm{~T}$.

\section{Concluding Remarks}

The well-known theoretical developments, such as the dynamical mean field theory (DMFT) $[7,8,35]$, may require in future a revisit of the problem of the quantum oscillations with a new perspective. Particularly, the development of DMFT and its cluster extensions provides new path to investigate strongly correlated systems; the DMFT study of superconductivity near the Mott transition establishes the remarkable coexistence of a superconducting gap, stemming from the anomalous self-energy, with a pseudogap stemming from the normal self-energy. This theory also leads to the generation of the Fermi arc behavior of the spectral function $[7,8]$. 
We conclude that the key input in our analysis is the Landau level split dispersion in (1); the chirality aspect perhaps plays a minor role as these oscillations are also possible in the pure d-density wave state [4]. We note that though the inclusion of the elastic scattering by impurities has led to a clearer understanding, of the Fermi surface topology in the presence of a magnetic field at the semiphenomenological level, the further examination of the single-particle excitation spectrum of the system in a fully self-consistent approximation framework is necessary to impart a comprehensive microscopic basis to the findings presented. Finally, we hope that our results, namely. the one relating to the reconstructed Fermi surface in the weak as well as the strong disorder regimes and the other to the electronic specific heat anomaly, will persuade researchers to look for them in the hole-doped cuprates. It must be added that the experimental observation of the latter is quite a difficult proposition, for the dominant phononic contribution is expected to overshadow the anomaly in the heat capacity measurements [36].

\section{References}

[1] C. Zhang, S. Tewari, V. M. Yakovenko, and S. Das Sarma, "Anomalous Nernst effect from a chiral d-density-wave state in underdoped cuprate superconductors," Physical Review B, vol. 78, no. 17, Article ID 174508, 2008.

[2] P. Kotetes and G. Varelogiannis, "Meissner effect without superconductivity from a chiral d-density wave," Physical Review B, vol. 78, no. 22, Article ID 220509, 2008.

[3] I. Dimov, P. Goswami, X. Jia, and S. Chakravarty, "Competing order, Fermi surface reconstruction, and quantum oscillations in underdoped high-temperature superconductors," Physical Review B, vol. 78, no. 13, Article ID 134529, 2008.

[4] S. Chakravarty and H.-Y. Kee, "Fermi pockets and quantum oscillations of the Hall coefficient in high-temperature superconductors," Proceedings of the National Academy of Sciences of the United States of America, vol. 105, no. 26, pp. 8835-8839, 2008.

[5] S. Tewari, C. Zhang, V. M. Yakovenko, and S. Das Sarma, "Time-reversal symmetry breaking by a $(\mathrm{d}+\mathrm{id})$ densitywave state in underdoped cuprate superconductors," Physical Review Letters, vol. 100, no. 21, Article ID 217004, 2008.

[6] K.-Y. Yang, T. M. Rice, and F.-C. Zhang, "Phenomenological theory of the pseudogap state," Physical Review B, vol. 73, no. 17, Article ID 174501, 2006.

[7] T. D. Stanescu and G. Kotliar, "Fermi arcs and hidden zeros of the Green function in the pseudogap state," Physical Review B, vol. 74, no. 12, Article ID 125110, 2006.

[8] M. Civelli, M. Capone, A. Georges et al., "Nodal-antinodal dichotomy and the two gaps of a superconducting doped mott insulator," Physical Review Letters, vol. 100, no. 4, Article ID 046402, 2008.

[9] R. K. Kaul, Y. B. Kim, S. Sachdev, and T. Senthil, "Algebraic charge liquids," Nature Physics, vol. 4, no. 1, pp. 28-31, 2008.

[10] V. Hinkov, D. Haug, B. Fauqué et al., "Electronic liquid crystal state in the high-temperature superconductor $\mathrm{YBa}_{2} \mathrm{Cu}_{3} \mathrm{O}_{6.45}$," Science, vol. 319, no. 5863, pp. 597-600, 2008.

[11] J. Xia, E. Schemm, G. Deutscher et al., "Polar kerr-effect measurements of the high-temperature $\mathrm{YBa}_{2} \mathrm{Cu}_{3} \mathrm{O}_{6+x}$ superconductor: evidence for broken symmetry near the pseudogap temperature," Physical Review Letters, vol. 100, no. 12, Article ID 127002, 2008.
[12] S. Riggs, J. Betts, S. Sebastian et al., "Abstract : L33-00004, 2009 APS March meeting(Unpublished)," G. S. Boebinger, private communication.

[13] N. Doiron-Leyraud, C. Proust, D. LeBoeuf et al., "Quantum oscillations and the Fermi surface in an underdoped high$\mathrm{T}_{c}$ superconductor," Nature, vol. 447, no. 7144, pp. 565-568, 2007.

[14] D. LeBoeuf, N. Doiron-Leyraud, J. Levallois et al., "Electron pockets in the Fermi surface of hole-doped high- $\mathrm{T}_{c}$ superconductors," Nature, vol. 450, no. 7169, pp. 533-536, 2007.

[15] A. F. Bangura, J. D. Fletcher, A. Carrington et al., "Small fermi surface pockets in underdoped high temperature superconductors: observation of Shubnikov-de Haas oscillations in $\mathrm{YBa}_{2} \mathrm{Cu}_{4} \mathrm{O}_{8}$," Physical Review Letters, vol. 100, no. 4, Article ID 047004, 2008.

[16] E. A. Yelland, J. Singleton, C. H. Mielke et al., "Quantum oscillations in the underdoped cuprate $\mathrm{YBa}_{2} \mathrm{Cu}_{4} \mathrm{O}_{8}$," Physical Review Letters, vol. 100, no. 4, Article ID 047003, 2008.

[17] C. Jaudet, D. Vignolles, A. Audouard et al., "De Haasvan Alphen oscillations in the underdoped high-temperature superconductor $\mathrm{YBa}_{2} \mathrm{Cu}_{3} \mathrm{O}_{6.5}$," Physical Review Letters, vol. 100, no. 18, Article ID 187005, 2008.

[18] S. E. Sebastian, N. Harrison, C. H. Mielke et al., "Spin-order driven fermi surface reconstruction revealed by quantum oscillations in an underdoped high $\mathrm{T}_{c}$ superconductor," Physical Review Letters, vol. 103, no. 25, Article ID 256405, 2009.

[19] S. E. Sebastian, N. Harrison, E. Palm et al., "A multicomponent Fermi surface in the vortex state of an underdoped high- $\mathrm{T}_{c}$ superconductor," Nature, vol. 454, no. 7201, pp. 200203, 2008.

[20] S. E. Sebastian, N. Harrison, P. A. Goddard et al., "Compensated electron and hole pockets in an underdoped high- $\mathrm{T}_{c}$ superconductor," Physical Review B, vol. 81, no. 21, Article ID 214524, 2010.

[21] R. Daou, J. Chang, D. Leboeuf et al., "Broken rotational symmetry in the pseudogap phase of a high- $\mathrm{T}_{c}$ superconductor," Nature, vol. 463, no. 7280, pp. 519-522, 2010.

[22] M. A. Hossain, J. D. F. Mottershead, D. Fournier et al., "In situ doping control of the surface of higherature superconductors," Nature Physics, vol. 4, no. 7, pp. 527-531, 2008.

[23] V. B. Zabolotnyy, S. V. Borisenko, A. A. Kordyuk et al., "Momentum and temperature dependence of renormalization effects in the high-temperature superconductor $\mathrm{YBa}_{2} \mathrm{Cu}_{3} \mathrm{O}_{7-\delta}$," Physical Review B, vol. 76, no. 6, Article ID $064519,2007$.

[24] C. Gros, B. Edegger, V. N. Muthukumar, and P. W. Anderson, "Determining the underlying Fermi surface of strongly correlated superconductors," Proceedings of the National Academy of Sciences of the United States of America, vol. 103, no. 39, pp. 14298-14301, 2006.

[25] P. W. Anderson, "Is there glue in the cuprate superconductors?” Science, vol. 316, p. 1705, 2007.

[26] A. Kanigel, M. R. Norman, M. Randeria et al., "Evolution of the pseudogap from Fermi arcs to the nodal liquid," Nature Physics, vol. 2, no. 7, pp. 447-451, 2006.

[27] K. M. Shen, F. Ronning, D. H. Lu et al., "Nodal quasiparticles and antinodal charge ordering in $\mathrm{Ca}_{2-x} \mathrm{Na}_{x} \mathrm{CuO}_{2} \mathrm{Cl}_{2}$," Science, vol. 307, no. 5711, pp. 901-904, 2005.

[28] A. Damascelli, Z. Hussain, and Z.-X. Shen, "Angle-resolved photoemission studies of the cuprate superconductors," Reviews of Modern Physics, vol. 75, no. 2, pp. 473-541, 2003. 
[29] J. Meng, W. Zhang, G. Liu et al., "Monotonic d-wave superconducting gap of the optimally doped $\mathrm{Bi}_{2} \mathrm{Sr}_{1.6} \mathrm{La}_{0.4} \mathrm{CuO}_{6}$ superconductor by laser-based angle-resolved photoemission spectroscopy," Physical Review B, vol. 79, no. 2, Article ID 024514, 2009.

[30] A. Hackl and M. Vojta, "Nernst-effect anisotropy as a sensitive probe of Fermi-surface distortions from electron-nematic order," Physical Review B, vol. 80, no. 22, Article ID 220514, 2009.

[31] J. M. Ziman, Principles of the Theory of Solids, Cambridge University Press, Cambridge, UK, 1972.

[32] G. Rickayzen, Green's Functions and Condensed Matter, chapter 4, Academic Press, London, UK, 1980.

[33] L. P. Kadanoff and G. Baym, Quantum Statistical Mechanics, Benzamin, New York, NY, USA, 1962.

[34] T. Pereg-Barnea, H. Weber, G. Refael, and M. Franz, "Quantum oscillations from Fermi arcs," Nature Physics, vol. 6, pp. 44-49, 2010.

[35] C. Weber, K. Haule, and G. Kotliar, "Strength of correlations in electron- and hole-doped cuprates," Nature Physics, vol. 6, no. 8, pp. 574-578, 2010.

[36] C. Meingast, A. Inaba, R. Heid et al., "Specific-heat of $\mathrm{YBa}_{2} \mathrm{Cu}_{3} \mathrm{O}_{x}$ up to $400 \mathrm{~K}$ : high-resolution adiabatic measurements and ab-initio LDA phonon calculations," Journal of the Physical Society of Japan, vol. 78, no. 7, Article ID 074706, 2009. 

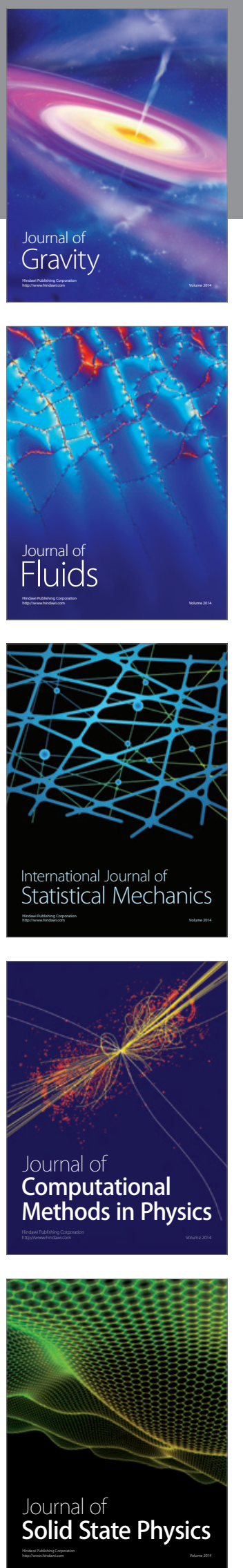

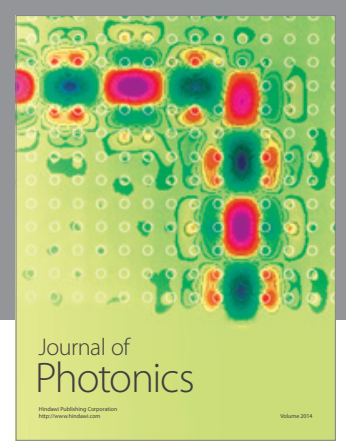

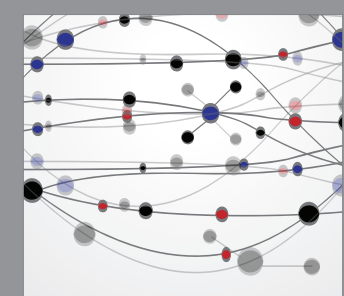

The Scientific World Journal
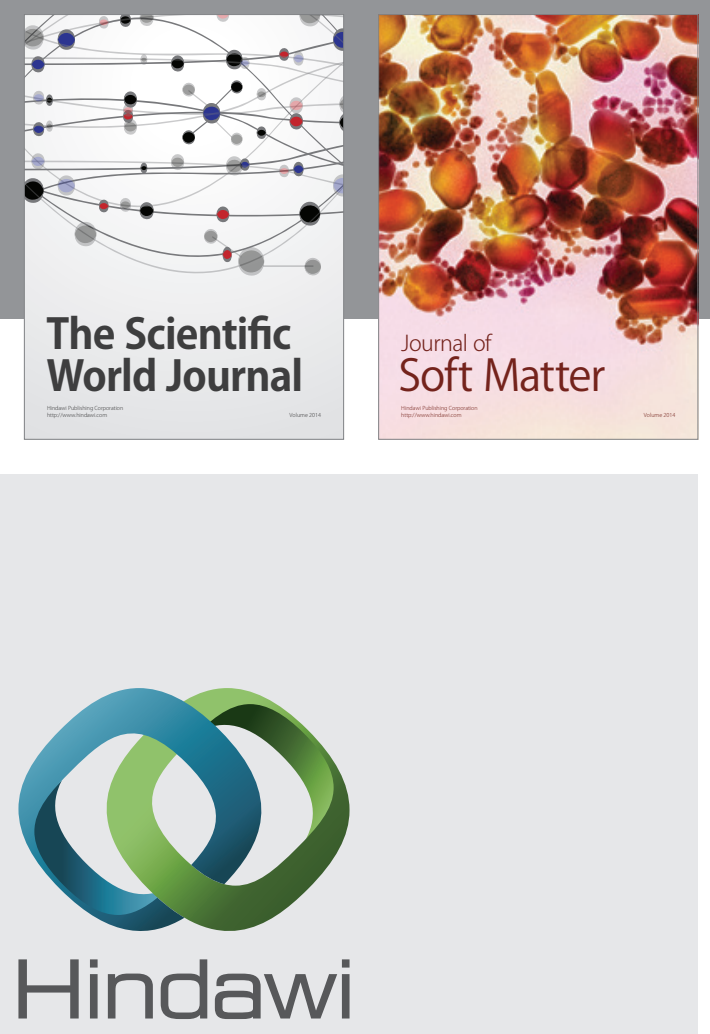

Submit your manuscripts at

http://www.hindawi.com
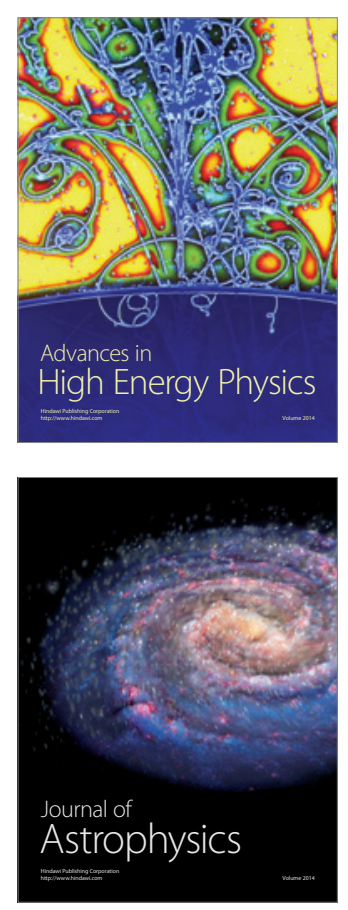
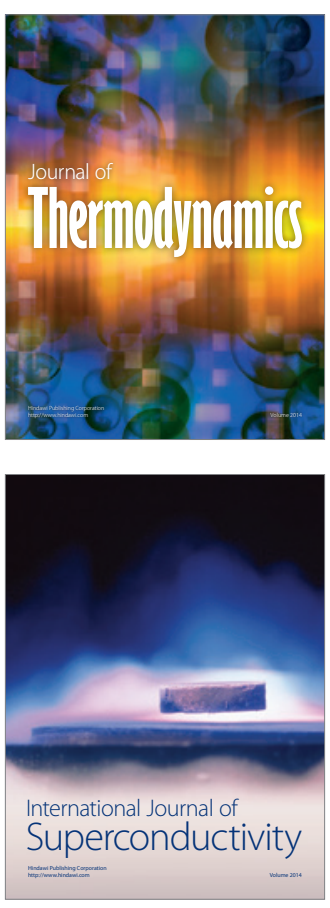
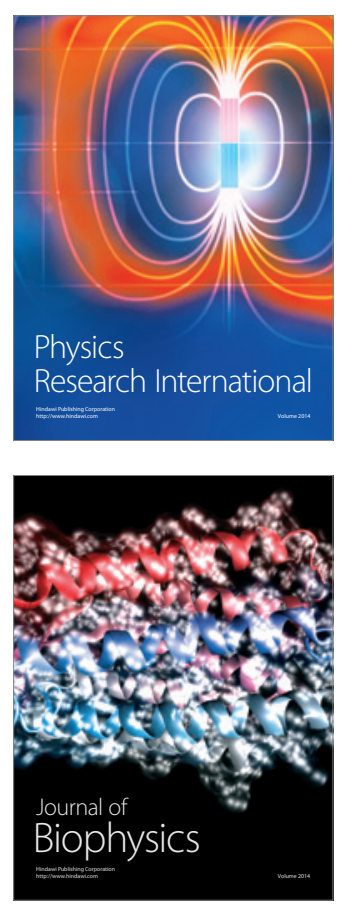
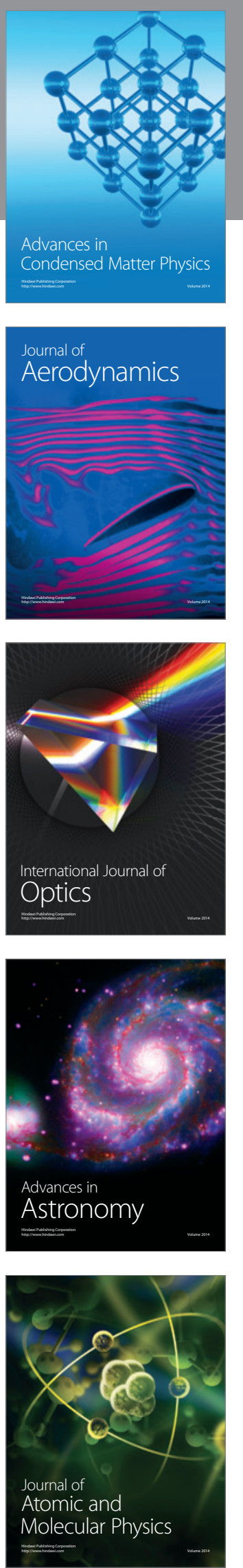